\title{
24. Outline of Universal Gyroscopic X-Ray Television Apparatus (Gyroscope) and its Clinical Value
}

\author{
By Toshio Kurokawa, M. J. A.,*) Tatuo SAITo,**) \\ and Shoji NishIYAMA***)
}

(Communicated Feb. 12, 1977)

I. Forewords. The stomach, intestine and colon have cubic complicated form respectively, and they are overlapped each other or have dead angles for an X-ray beam.

On the other hand, the X-ray beam of conventional equipment is limited directionally.

Consequently, it is difficult to take completely separated images with the conventional equipment. Especially, even in these days when double contrast radiography of stomach and colon becomes popular, it is more difficult to take barium filled and double contrast images of every region of stomach and colon.

Then, we have contrived a new X-ray apparatus and called it Universal Gyroscopic X-Ray Television Apparatus (petname: Gyroscope). We intend to report the outline of this apparatus and state its clinical value with presentation of some cases.

II. Outline of Gyroscope. Fig. 1 shows the outside view of Gyroscope. Features of Gyroscope are as follows :

A) Tilting: A fluoroscopic table can be tilted backward from upright to Trendelenburg position (till $-90^{\circ}$ from horizontal).

B) Hain Rotation System: A patient, an X-ray tube and a spot-shot filming device can be rotated more than $360^{\circ}$ in a single unit toward both sides in every angle of tilt of the table. Figs. 2 and 4 show this performance.

C) Patient Rolling System: In addition to the Main Rotation System, patient alone can be rotated toward both sides till $90^{\circ}$ (total $180^{\circ}$ ). Therefore, radiograms in right and left anterior oblique positions can be taken as same as conventional method. Figs. 3 and 5 show this performance.

D) Beam Rotation System: In every position, an X-ray tube can be rotated together with a spot-shot filming device around a Tokyo.

*) Cancer Institute Hospital (Japanese Foundation for Cancer Research),

**) Department of Clinical Cancer Chemotherapy, The Research Institute for Tuberculosis, Leprosy and Cancer, Tohoku University, Sendai.

***) Nishiyama's Gastrointestinal Clinic, Hachinohe. 

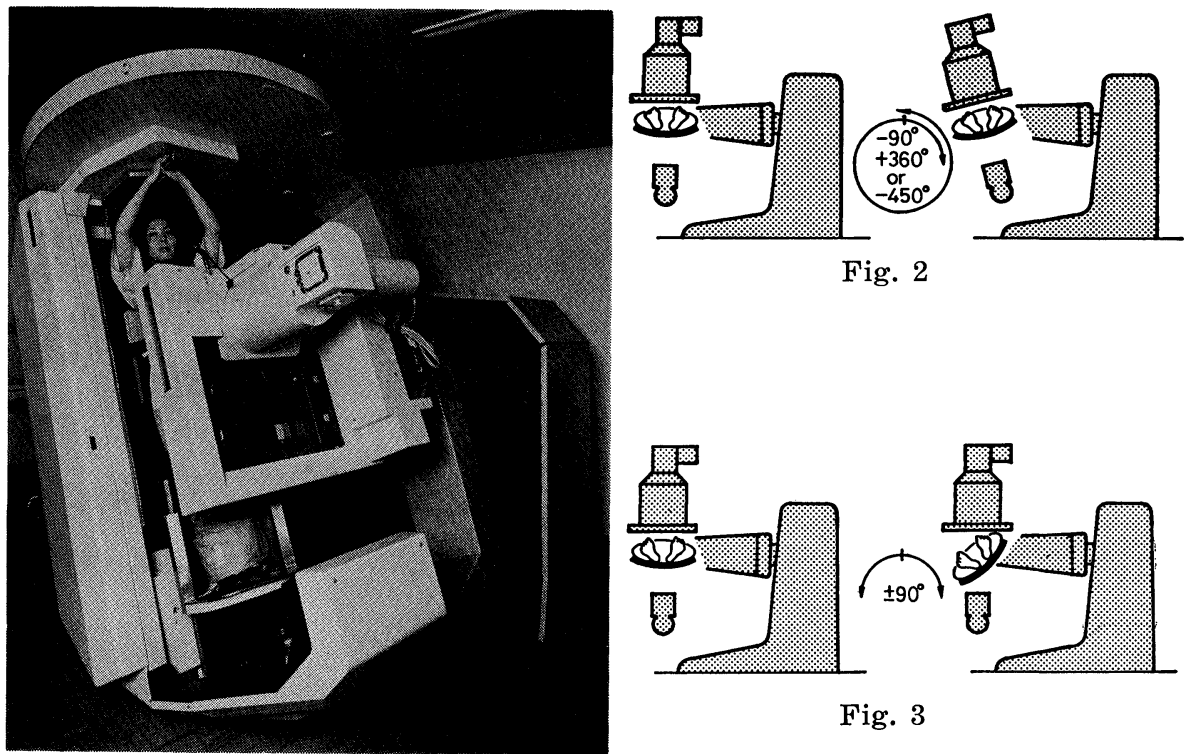

Fig. 2

Fig. 1

Fig. 1. Universal Gyroscopic X-Ray Television Apparatus (petname: Gyroscope).

Fig. 2. Main Rotation System. Patient can be rotated together with an Xray tube and a spot-shot filming device as one unit.

Fig. 3. Patient Rolling System. Patient alone can be rolled toward both sides.

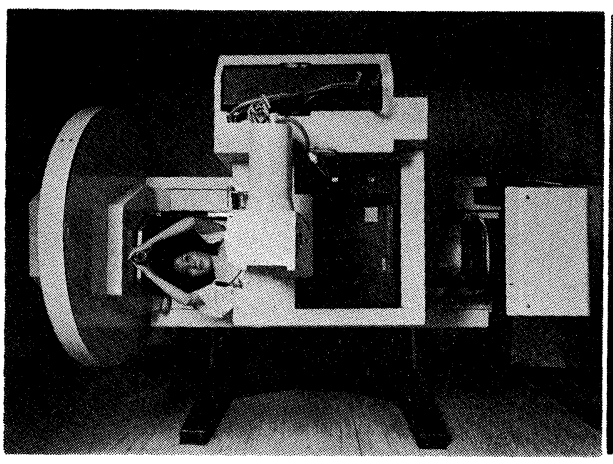

Fig. 4

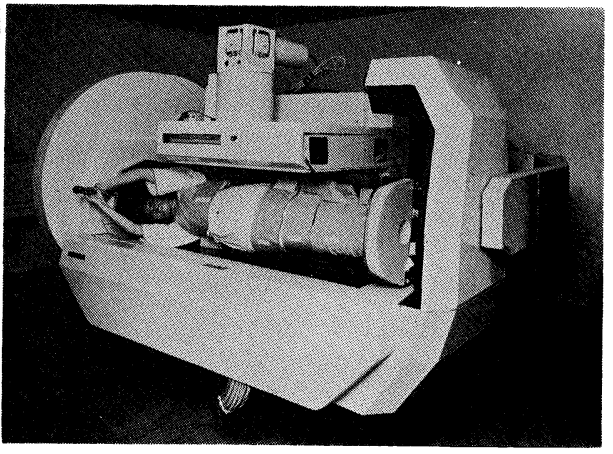

Fig. 5

Fig. 4. Dorso-ventral radiography in right recumbent position. Patient is supported by the special Fastening Band.

Fig. 5. Lateral radiography by making use of the Patient Rolling System or Beam Rotation System. Of course, this procedure is possible in supine, prone and other positions. A part of the Fastening Band can be seen.

patient toward both sides till $90^{\circ}$. Fig. 5 shows this performance.

E) Fastening Band: As mentioned above, when a patient is rotated by the Main Rotation and Patient Rolling Systems, he has 
to be immobilized by a special Fastening Band to prevent him from falling off. This band can fit the contour of his body regardless of whether he is fat or thin, short or tall. A part of the band can be seen in Figs. 1 and 5.

F) Stereoradiography: Stereoscopic fluorography is possible by using of the Patient Rolling or Beam Rotation System.

G) Others: Accessories and others are omitted.

III. Clinical cases. Some pictures taken by this apparatus are shown below.

Case 1. Scar at the anterior wall of antrum. Fig. 6 is a picture of benign ulcer at the anterior wall of antrum. We toiled to take this picture by making use of conventional equipment. Fig. 7 is a double contrast picture of the same patient which was taken by using Gyroscope after 7 months.

At first, the gastric anterior wall was made up with barium in prone position, and this picture was taken in left recumbent position rotated from the prone position by the Main Rotation System. The ulcer which had scarred already and convergence of folds can be seen in this picture.

Thus, even a fine change of gastric anterior wall can be demonstrated easily by Gyroscope.

Case 2. Early gastric cancer. Fig. 8 is a double contrast picture of stomach in supine position. In this picture, a depression is illustrated at the posterior wall of gastric corpus, but each nature and forms of folds surrounding it are not illustrated completely, because barium meal stagnated at the corpus and could not be removed by all means. Therefore, it was impossible to diagnose whether the depression was benign or malignant.

Fig. 9 is the picture of the same patient. This picture was taken in right recumbent position by a dorso-ventral projection. Barium meal which stagnated and covered folds surrounding the depression was removed in this position that was rotated from the supine position by the Main Rotation System. These folds were demonstrated clearly in this picture, therefore a qualitative diagnosis was possible without doubt. This case was diagnosed as early cancer and confirmed by a surgical operation.

Thus, barium meal and air can be moved at will by Gyroscope. This is very important in case of double contrast radiography.

IV. Summary. We contrived and published Universal Gyroscopic X-Ray Television Apparatus, that is Gyroscope.

As mentioned above, Gyroscope has many features; the Fastening Band, Table-Tilting, Main Rotation, Patient Rolling, Beam Rotation Systems and others. 


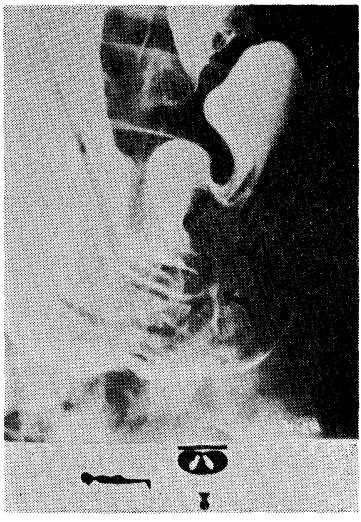

Fig. 6

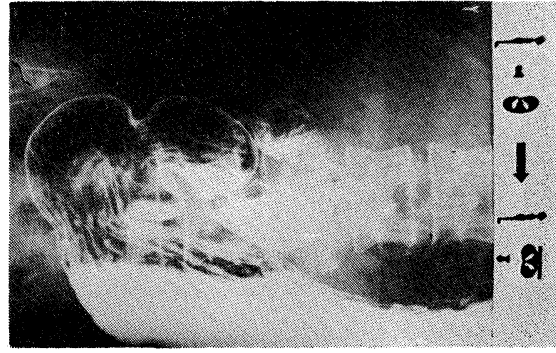

Fig. 7

Fig. 6. Ulcer (arrow) at the anterior wall of antrum. The picture was taken by a conventional equipment.

Fig. 7. Scar (arrow) at the same region. (The same patient.) The picture was taken after 7 months by Gyroscope. It was taken in left recumbent position which was rotated from the prone position by the Main Rotation System.

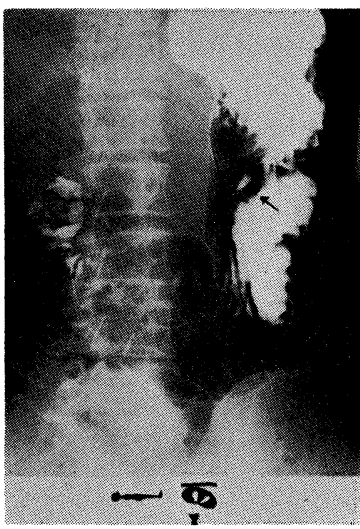

Fig. 8

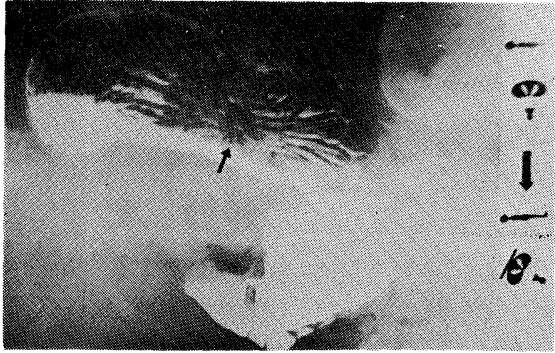

Fig. 9

Fig. 8. Double contrast picture in supine position. The fleck of barium (arrow) can be seen and suggests a depression. But it is impossible to diagnose whether it is malignant or benign.

Fig. 9. Gastric picture of the same patient. The picture was taken in right recumbent position by a dorso-ventral projection. The depression (arrow) was diagnosed as early gastric cancer.

Therefore, Gyroscope is able to rotate like as planetarium, and a patient can be taken all sorts of positions and also exposed from many directions. These things are necessary and useful for the double contrast radiography of gastrointestinal tract and others.

With presentation of cases mentioned above, we stated the clinical usefulness of Gyroscope in addition to a usual method. 